\title{
Nutrientes na água para irrigação de arroz na Região Sul do Rio Grande do Sul, Brasil
}

\author{
Nutrients in the water for irrigation of rice in the South region of the State of Rio \\ Grande do Sul, Brazil
}

\author{
Marcelo Diel ${ }^{\mathrm{I}}$ Rosa Maria Vargas CastilhosII Rogério Oliveira de Sousa ${ }^{\mathrm{II}}$ \\ Ledemar CarlosValh"I João Baptista da Silva ${ }^{\text {II }}$
}

\section{RESUMO}

A cultura do arroz irrigado apresenta baixa resposta à adubação em comparação com as culturas de sequeiro, cultivadas em solos com as mesmas características de fertilidade. Isto é causado, em parte, pelas transformações físico-químicas que ocorrem no solo devido ao alagamento, favorecendo a disponibilização de nutrientes. Outro fator que também pode estar contribuindo para a baixa resposta do arroz à adubação é o aporte de nutrientes para a lavoura, pela água utilizada na irrigação. Com o objetivo de quantificar os teores de nutrientes nas águas dos principais mananciais utilizados para irrigação de lavouras de arroz da Região Sul do Estado do Rio Grande do Sul, Brasil, bem como estimar a sua possível contribuição para a nutrição desta cultura, foram coletadas sessenta amostras, sendo vinte de cada tipo de manancial - açudes, lagoas e rios. Nestas foram determinados os teores de $N$-amônio, $N$-nitrato, fósforo $(P)$, potássio $(K)$, cálcio (Ca), magnésio $(\mathrm{Mg})$, sódio $(\mathrm{Na})$, zinco $(\mathrm{Zn})$, ferro $(\mathrm{Fe})$, manganês (Mn), silício (Si), pH e condutividade elétrica (CE). $\mathrm{Na}$ média, os elementos presentes nas águas de açudes, lagoas e rios utilizados para irrigação de arroz e na Região Sul do Rio Grande do Sul decresceram na seqüência $\mathrm{Na}>\mathrm{Ca}>\mathrm{Si}>\mathrm{Fe}>$ $M g>K>M n>N>Z n>P$. Os teores médios diferiram entre mananciais, sendo mais elevados $N$, Fe e Si nos rios, do que nas lagoas; Ca e Mg nas lagoas, do que nos açudes; e Fe nos açudes e rios, do que nas lagoas. Os aportes de nutrientes estimados através de suas concentrações nas águas de irrigação mostraram que essas quantidades correspondem ao que, em média, é acumulado nas plantas de $\mathrm{Ca}$, $\mathrm{Fe}$, Si e $\mathrm{Na}$ e até $28 \%$ de $\mathrm{Mg}$ e $12 \%$ de $\mathrm{K}$.

Palavras-chave: lagoas, açudes, rios, Oryza sativa.

\section{ABSTRACT}

The flooded rice crop presents low response to fertilization when compared to dry land crops, cultivated in soils with same characteristics of fertility. This is caused, in part, by physical and chemical changes that take place in the soil due to flooding, increasing nutrients availability. Another factor that also can cause the low response of rice crops to fertilization is nutrients supply through the water used in the irrigation. This paper was aimed at quantifing nutrients concentration in the irrigation waters used in the Southern region of the State of Rio Grande do Sul, Brazil, as well as estimating its possible contribution to rice plant nutrition. Sixty samples of waters were collected from dams, lagoons and rivers, twenty in each source. It was determined $\mathrm{N}$-ammonium, $\mathrm{N}$ nitrate, phosphorus ( $P)$, potassium (K), calcium (Ca), magnesium ( $\mathrm{Mg})$, sodium $(\mathrm{Na})$, zinc $(\mathrm{Zn})$, iron $(\mathrm{Fe})$, manganese $(\mathrm{Mn})$, silicon ( $\mathrm{Si}$ ) content, $\mathrm{pH}$ and electrical conductivity (CE). The elements concentration in the irrigation waters decreased in the following order $\mathrm{Na}>\mathrm{Ca}>\mathrm{Si}>\mathrm{Fe}>\mathrm{Mg}>\mathrm{K}>\mathrm{Mn}>\mathrm{N}>$ $\mathrm{Zn}>P$. The average elements contents were different between irrigation sources: $N$ and Si content in rivers were higher than those in the lagoons; $\mathrm{Ca}$ and $\mathrm{Mg}$ were more concentrated in lagoons than in dams; Fe was more concentrated in dams and rivers compared to the lagoons. The estimated nutrient contribution by irrigation waters can correspond to all of the calcium, iron, silicon and sodium, and up to $28 \%$ of magnesium and $12 \%$ of potassium uptake, in average, by rice plants.

Key words: dam, lagoon, rivers, Oryza sativa.

\section{INTRODUÇÃO}

Uma característica da cultura do arroz irrigado é sua baixa resposta à adubação em comparação com as culturas de sequeiro. Isto pode ser causado, em parte, pelas transformações físicoquímicas que ocorrem no solo devido ao alagamento e que favorecem a disponibilização de nutrientes, como

\footnotetext{
'Programa de Pós-graduação em Agronomia, Área de Concentração em Solos, Universidade Federal de Pelotas (UFPel), Pelotas, RS, Brasil.

"Departamento de Solos, Faculdade de Agronomia Eliseu Maciel (FAEM), CP 354, 96010-900, Pelotas, RS, Brasil. E-mail: rosamvc@ufpel.edu.br. Autor para correspondência.

IIIInstituto de Física e Matemática (IFM), (UFPel), Pelotas, RS, Brasil.
} 
também pelo aporte de nutrientes para a lavoura, através da água utilizada na irrigação (VAHL, 2004). No Estado do Rio Grande do Sul, a utilização total de água para as lavouras de arroz irrigado, com base na área plantada, é estimada entre 8.000 e $15.500 \mathrm{~m}^{3}$ ha $^{-1}$ por safra, e os principais mananciais fontes para captação de água para irrigação são rios, açudes e lagoas (GOMES et al., 2004). Mesmo em baixas concentrações, a presença de nutrientes nestes mananciais pode vir a ser significativa na nutrição da cultura, em razão do elevado volume de água utilizado nas lavouras.

Os teores de nutrientes e outros parâmetros da água de irrigação das lavouras de arroz foram avaliados por diversos autores, em diferentes mananciais hídricos do RS e de $\mathrm{SC}$, e os resultados indicam que há ampla variação de concentração (MACEDO et al., 2001a e b; FURTADO et al., 2001; DESCHAMPS et al., 2003a; MACEDO et al. 2003a, b, c; MATTOS, 2004; MACEDO et al., 2005). A diversidade que se observa na composição química das águas está associada à geologia local, aos eventos da natureza como chuvas ou secas e às atividades antropogênicas que, na atualidade, são grandes transformadoras da qualidade das águas superficiais (DECHAMPS et al., 2003b).

Quanto à possível contribuição das águas de irrigação para a nutrição do arroz, alguns trabalhos destacam as concentrações de $\mathrm{K}, \mathrm{Ca}$ e $\mathrm{Mg}$ nas águas do Rio Gravataí (Cachoeirinha, RS), reconhecidamente rico em nutrientes devido à alta carga orgânica e industrial que recebe (MACEDO et al., 2001a e b; MACEDO et al. 2003c). Esses autores estimaram uma contribuição das águas deste rio para a nutrição do arroz equivalente a $80 \mathrm{~kg} \mathrm{ha}^{-1}$ de nitrogênio; $1,6 \mathrm{~kg} \mathrm{ha}^{-1}$ de $\mathrm{P}_{2} \mathrm{O}_{5}$ e $58 \mathrm{~kg} \mathrm{ha}^{-1}$ de $\mathrm{K}_{2} \mathrm{O}$. Em outro estudo, GENRO JR. et al. (2005), comparando o aporte de nutrientes pela água de irrigação do Rio Gravataí com a do reservatório da barragem do Capané (Cachoeira do Sul, RS), em três fases da cultura do arroz, constataram que o aporte de nutrientes foi maior no Rio Gravataí e ocorreu no período do florescimento pleno, sendo equivalente a $36 \mathrm{~kg} \mathrm{ha}^{-1} \mathrm{de} \mathrm{N}, 2,1 \mathrm{~kg} \mathrm{ha}^{-1} \mathrm{de}_{2} \mathrm{O}_{5}$ e $17 \mathrm{~kg}$ ha $^{-1}$ de $\mathrm{K}_{2} \mathrm{O}$.

Alguns trabalhos também têm evidenciado que a lavoura de arroz irrigado pode atuar como filtro biológico, retirando nutrientes do meio hídrico eutrofizado através da sua utilização pelas plantas, diminuindo assim a concentração de nutrientes na água de drenagem, em comparação com a água que entra na lavoura (FURTADO et al., 2001; MACEDO et al., 2003c GENRO JR. et al., 2005).

No RS, poucos são os trabalhos analisando a composição das águas de irrigação destinadas às lavouras de arroze, especialmente para a Região Sul do Estado, inexistem pesquisas com este propósito. Assim, o presente trabalho teve por objetivo quantificar os teores de nutrientes e outros parâmetros nas águas dos principais mananciais, fontes de captação de águas para irrigação de lavouras de arroz da Região Sul do Rio Grande do Sul, bem como estimar a sua possível contribuição para a nutrição desta cultura.

\section{MATERIAL E MÉTODOS}

Durante os meses de janeiro e fevereiro, na safra agrícola de 2003/2004, na Região Sul do Rio Grande do Sul, Brasil, foram coletadas sessenta amostras de águas, sendo vinte amostras em cada tipo de manancial (lagoas, açudes e rios), principais fontes de captação de água para irrigação de lavouras de arroz. Em algumas das fontes, foram coletadas amostras em mais de um local, em razão da sua importância em extensão e em volume de água utilizada na irrigação. As fontes amostradas e a quantidade de locais amostrados em cada uma delas (entre parênteses) foram:

Açudes: EMBRAPA - Barragem do Lino (1), Centro Agropecuário da Palma - UFPEL (2), Guarita Bretanhas (1), Barragem do Chasqueiro (1), Granja Teodoro (2), Jaguarão - ao lado da BR 116 (1), Bento Gonçalves - BR 116(1), Santa Rita (1), Butiá (1), Barragem dos Órfãos (1), Granja Capão Seco (1), Domingos Petrolini (2), Nelson Vendt (1), Granja Três Capões (1), Retiro (1), Barragem do Duro - Arroio Duro (1) e Barragem Luiz Rechsteiner (1);

Lagoas: Patos (1), Mangueira (3), Caiubá (2), Mirim (6), Pequena (1) e Canal São Gonçalo (7) que, por unir as lagoas dos Patos e Mirim, foi considerado como lagoa;

Rios: Jaguarão (1), Piratini (1), Arroio Grande (2), Camaquã (1) Pavão (1), Juncal (1), Bretanhas (1), Capivaras (1), Moreira (1), Pelotas (2), Corrientes (2), Velhaco (2), Jacaré (1), Santa Rita (1), Contagem (1) e Retiro (1).

Para a coleta das amostras, utilizou-se uma garrafa PET com capacidade para dois litros, perfurada da extremidade superior até o centro, à qual foi amarrada uma corda, para facilitar o seu lançamento e retirada da água, e pesos de chumbo para assegurar que afundasse na vertical. A água de cada amostragem foi transferida para duas garrafas plásticas de $500 \mathrm{~mL}$, uma delas já contendo $2 \mathrm{~mL}$ de $\mathrm{H}_{2} \mathrm{SO}_{4} 2,5 \mathrm{~mol} \mathrm{~L}^{-1}$, para a acidificação da amostra $(\mathrm{pH}<2,0)$, evitando-se com isto perdas de nitrogênio. As garrafas foram conservadas em caixas de isopor, com gelo, durante o seu transporte até o Laboratório de Análises. Neste, as amostras acidificadas foram armazenadas em geladeira e, um dia 
após cada coleta, analisadas quanto aos teores de amônio e nitrato, pelo método de destilação por arraste de vapor. Também de imediato ou, no máximo no dia seguinte à coleta, $\mathrm{o} \mathrm{pH}$ e a condutividade elétrica foram determinados em uma alíquota das amostras nãoacidificadas, sem nenhum tratamento prévio (TEDESCO et al., 1995). Após isso, o restante destas amostras foi filtrado em papel JP no 42, e armazenado a uma temperatura inferior a $4^{\circ} \mathrm{C}$, para análises posteriores.

Para as determinações de fósforo, potássio, cálcio, magnésio, sódio, zinco, ferro e manganês, uma alíquota de $100 \mathrm{~mL}$ das amostras filtradas foi clarificada com $2 \mathrm{~mL}$ de $\mathrm{HCl} 50 \%$. O Na e o $\mathrm{K}$ foram determinados por fotometria de chama. Os teores de $\mathrm{Ca}, \mathrm{Mg}, \mathrm{Zn}, \mathrm{Mn}$ e $\mathrm{Fe}$ foram quantificados por espectrofotometria de absorção atômica. A determinação do $\mathrm{P}$ foi feita por espectrofotometria. A determinação do teor de silício na água foi feita pelo método descrito por KORNDÖRFER et al. (1999).

Os resultados foram submetidos à análise da variação e ao teste de Duncan, a 5\% de probabilidade, para a comparação das médias entre mananciais. Foi feita análise estatística descritiva para cada um dos mananciais - açudes, lagoas e rios obtendo-se a distribuição de freqüências, valores mínimos e máximos, coeficiente de variação, mediana, que melhor representa a tendência central das observações quando estas não seguem uma distribuição normal (o que foi constatado para a maioria dos parâmetros), e o intervalo de confiança da média, ao nível de $5 \%$, que assegura $95 \%$ de probabilidade das estimativas de um parâmetro situar-se dentro do referido intervalo, em amostragens subseqüentes. $\mathrm{O}$ programa utilizado foi o SANEST - Sistema de Análise Estatística. Departamento de Matemática, Estatística e Computação - IFM - UFPEL (ZONTA \& MACHADO, 1984).

\section{RESULTADOS E DISCUSSÃO}

Os resultados da composição química das águas de açudes, rios e lagoas são apresentados na tabela 1. Nos açudes, apresentaram a maior amplitude de variação: $\mathrm{K}, \mathrm{Ca}, \mathrm{Mg}$, Na, Fe, Mn e Si, com destaque para o $\mathrm{Na}$, que teve o maior valor $\left(25330 \mu \mathrm{g} \mathrm{L}^{-1}\right)$. Este fato deve também ter contribuído para a elevada amplitude da condutividade elétrica, de $413 \mu \mathrm{S} \mathrm{cm}^{-1}$, encontrada nos açudes. O Mn, além de alta amplitude, apresentou o maior coeficiente de variação $(\mathrm{CV}=173 \%)$ dentre os parâmetros analisados. Os coeficientes de variação de $\mathrm{N}-\mathrm{NO}_{3}, \mathrm{P}, \mathrm{Na}$ e $\mathrm{CE}$ também foram altos $(>100 \%)$, indicando grande variabilidade destes parâmetros dentro do mesmo tipo de manancial. O pH foi o que menos variou, com menor amplitude e menor CV. Os demais parâmetros analisados mantiveram-se em uma faixa intermediária de variação.

Observando-se os dados da mediana, constata-se que ocorreram em maiores concentrações nas águas dos açudes, em ordem decrescente: $\mathrm{Si}>\mathrm{Ca}>$ $\mathrm{Na}>\mathrm{Fe}>\mathrm{Mg}>\mathrm{K}$ e, em menores concentrações, o $\mathrm{P}$ e o $\mathrm{Zn}$. As concentrações de $\mathrm{Ca}, \mathrm{Fe}, \mathrm{Mg}, \mathrm{K}$ e P observadas nos açudes da Região Sul do RS são semelhantes para o Ca e menores para os demais elementos do que as encontradas por Macedo et al. (2001b), ao efetuarem o levantamento exploratório da composição química das águas dos açudes Barragem do Capané e Barragem do Arroio Duro; entretanto, as concentrações médias de $\mathrm{Zn}$ e de $\mathrm{Na}$ foram maiores. A maior concentração de $\mathrm{Na}$ nas águas dos açudes da Região Sul do RS pode estar relacionada ao fato de, nesta região, predominarem Planossolos da unidade de mapeamento Pelotas, classificados como Planossolos Hidromórficos eutróficos solódicos (PINTO et al., 2004).

Nas lagoas (Tabela 1), o sódio foi o elemento que mais variou, apresentando a maior amplitude e o maior coeficiente de variação. Isto pode ser justificado pelas entradas periódicas de água do mar em algumas delas, principalmente na Lagoa dos Patos, contribuindo para a elevação dos valores de $\mathrm{Na}$, como também para a alta condutividade elétrica encontrada em algumas amostras. Além do $\mathrm{Na}$, o $\mathrm{K}$, o $\mathrm{Ca}$, o $\mathrm{Mg}$, o Fe e o $\mathrm{Si}$ tiveram amplitudes elevadas. Os coeficientes de variação da $\mathrm{CE}, \mathrm{N}_{-} \mathrm{NO}_{3}, \mathrm{P}$ e $\mathrm{Mn}$ também foram altos nas lagoas. $\mathrm{O}$ elevado $\mathrm{CV}$ do $\mathrm{N}-\mathrm{NO}_{3}$ pode ser explicado pelo fato de que algumas das lagoas utilizadas para a irrigação da cultura do arroz na Região Sul localizam-se em regiões mais densamente povoadas, aumentando as chances de contaminação destas por esgoto urbano, que, associado aos dejetos de animais criados na região, contribuem para a elevação dos teores de nitrato em algumas fontes (MATTOS, 2004).

Com base na concentração estimada pela mediana, ocorreram em maior concentração nas lagoas, em ordem decrescente: $\mathrm{Ca}>\mathrm{Na}>\mathrm{Si}>\mathrm{Fe}>\mathrm{Mg}>\mathrm{Ke}$, em menor concentração, o $\mathrm{P}$ e o N-NO . Em comparação à composição média de águas de lagoas de outras regiões do Estado, os teores de $\mathrm{P}$ e Na encontrados nas lagoas da Região Sul do RS foram semelhantes; os de $\mathrm{Ca}, \mathrm{K}$, $\mathrm{Mg}, \mathrm{Fe}, \mathrm{N}\left(\mathrm{NH}_{4}+\mathrm{NO}_{3}\right)$ foram menores, enquanto os de $\mathrm{Zn}$ e Mn foram maiores (MACEDO et al., 2001b).

Nos rios (Tabela 1), as maiores amplitudes ocorreram para Ca $\left(12230 \mu \mathrm{g} \mathrm{L}^{-1}\right), \mathrm{Na}\left(8960 \mu \mathrm{g} \mathrm{L} \mathrm{L}^{-1}\right)$ e Si $\left(9890 \mu \mathrm{g} \mathrm{L}^{-1}\right) . \mathrm{O} \mathrm{pH}$ teve o menor coeficiente de variação e o fósforo o maior. Os baixos valores analíticos obtidos para as concentrações de $\mathrm{P}$ nas águas dos rios podem 
Tabela 1 - Valores mínimos e máximos, coeficiente de variação (CV), mediana, intervalo de confiança (IC) a 5\%, com limite inferior (LI) e limite superior (LS) dos parâmetros: $\mathrm{pH}$, condutividade elétrica $\left(\mathrm{CE}, \mu \mathrm{S} \mathrm{cm}^{-1}\right)$ e nutrientes $\left.(\mu \mathrm{g} \mathrm{L})^{-1}\right)$, nas água de açudes, lagoas e rios da Região Sul do RS.

\begin{tabular}{|c|c|c|c|c|c|c|}
\hline \multirow{2}{*}{ Parâmetros } & \multirow{2}{*}{$\frac{\text { Valor }}{\text { mínimo }}$} & \multirow{2}{*}{$\frac{\text { Valor }}{\text { máximo }}$} & \multirow{2}{*}{ CV (\%) } & \multirow{2}{*}{ Mediana } & \multicolumn{2}{|c|}{ IC 5\% } \\
\hline & & & & & LI & LS \\
\hline \multicolumn{7}{|c|}{ Açudes } \\
\hline $\mathrm{pH}$ & 6,0 & 8,5 & 9 & 6,6 & 6,4 & 7,0 \\
\hline $\mathrm{CE}$ & 29 & 442 & 116 & 50 & 36 & 122 \\
\hline $\mathrm{N}-\mathrm{NH}_{4}$ & 16 & 267 & 62 & 81 & 70 & 127 \\
\hline $\mathrm{N}-\mathrm{NO}_{3}$ & 6,9 & 359 & 107 & 53 & 47 & 142 \\
\hline $\mathrm{P}$ & 1,7 & 89 & 105 & 16 & 14 & 40 \\
\hline $\mathrm{K}$ & 190 & 4100 & 99 & 635 & 463 & 1265 \\
\hline $\mathrm{Ca}$ & 490 & 9900 & 62 & 2650 & 2224 & 4043 \\
\hline $\mathrm{Mg}$ & 440 & 2910 & 62 & 750 & 665 & 1203 \\
\hline $\mathrm{Na}$ & 1470 & 26800 & 124 & 2940 & 1890 & 7071 \\
\hline $\mathrm{Zn}$ & 33 & 155 & 50 & 60 & 64 & 103 \\
\hline $\mathrm{Fe}$ & 750 & 3380 & 38 & 1560 & 1362 & 1950 \\
\hline $\mathrm{Mn}$ & 63 & 2225 & 173 & 121 & 55 & 524 \\
\hline $\mathrm{Si}$ & 150 & 10010 & 72 & 3350 & 2514 & 5050 \\
\hline \multicolumn{7}{|c|}{ Lagoas } \\
\hline $\mathrm{pH}$ & 6,1 & 8,6 & 8,6 & 6,9 & 6,7 & 7,3 \\
\hline $\mathrm{CE}$ & 82 & 784 & 89 & 136 & 102 & 246 \\
\hline $\mathrm{N}-\mathrm{NH}_{4}$ & 32 & 147 & 45 & 63 & 56 & 85 \\
\hline $\mathrm{N}-\mathrm{NO}_{3}$ & 12 & 274 & 98 & 42 & 38 & 101 \\
\hline $\mathrm{P}$ & 3,4 & 128 & 91 & 25 & 20 & 50 \\
\hline K & 470 & 2920 & 48 & 910 & 866 & 1370 \\
\hline $\mathrm{Ca}$ & 2820 & 20410 & 53 & 7300 & 6133 & 10130 \\
\hline $\mathrm{Mg}$ & 790 & 3340 & 38 & 1360 & 1250 & 1782 \\
\hline $\mathrm{Na}$ & 2860 & 49570 & 130 & 5100 & 3064 & 12580 \\
\hline $\mathrm{Zn}$ & 37 & 155 & 42 & 93 & 71 & 106 \\
\hline $\mathrm{Fe}$ & 850 & 1840 & 15 & 1370 & 1240 & 1423 \\
\hline $\mathrm{Mn}$ & 31 & 937 & 101 & 141 & 98 & 272 \\
\hline $\mathrm{Si}$ & 520 & 7680 & 69 & 2500 & 1875 & 3670 \\
\hline \multicolumn{7}{|c|}{ Rios } \\
\hline $\mathrm{pH}$ & 6,2 & 7,8 & 7,3 & 6,5 & 6,5 & 7,0 \\
\hline $\mathrm{CE}$ & 63 & 180 & 32 & 83 & 79 & 107 \\
\hline $\mathrm{N}-\mathrm{NH}_{4}$ & 18 & 476 & 77 & 104 & 92 & 194 \\
\hline $\mathrm{N}-\mathrm{NO}_{3}$ & 25 & 421 & 74 & 130 & 87 & 179 \\
\hline $\mathrm{P}$ & 3,9 & 398 & 155 & 22 & 16 & 103 \\
\hline $\mathrm{K}$ & 250 & 3410 & 73 & 700 & 596 & 1216 \\
\hline $\mathrm{Ca}$ & 2220 & 14450 & 52 & 5090 & 4390 & 7197 \\
\hline $\mathrm{Mg}$ & 670 & 2140 & 32 & 1170 & 1040 & 1403 \\
\hline $\mathrm{Na}$ & 2580 & 11540 & 43 & 4585 & 3984 & 5963 \\
\hline $\mathrm{Zn}$ & 37 & 155 & 59 & 48 & 47 & 84 \\
\hline $\mathrm{Fe}$ & 1160 & 2630 & 23 & 1530 & 1455 & 1805 \\
\hline $\mathrm{Mn}$ & 63 & 422 & 52 & 195 & 165 & 271 \\
\hline $\mathrm{Si}$ & 560 & 10450 & 54 & 5010 & 3770 & 6325 \\
\hline
\end{tabular}

explicar o alto CV (155\%) deste nutriente. Com base na mediana, os elementos em maiores concentrações nas águas dos rios, em ordem decrescente, foram $\mathrm{Ca}>\mathrm{Si}>$ $\mathrm{Na}>\mathrm{Fe}>\mathrm{Mg}>\mathrm{K}$ e os em menores concentrações foram $\mathrm{Zn}$ e P. Os valores de $\mathrm{pH}, \mathrm{Ca}, \mathrm{Na}$ e P nas águas dos rios da Região Sul do RS foram maiores do que os encontrados em outros rios do RS; entretanto, as concentrações médias de $\mathrm{N}-\mathrm{NH}_{4}, \mathrm{~N}-\mathrm{NO}_{3}, \mathrm{Mg}$, e K foram menores (MARCHEZAN et al., 2002).

Comparando-se os coeficientes de variação dos três mananciais (Tabela 1), observa-se, de modo geral, que as águas dos rios apresentaram menor 
variação nos parâmetros analisados que as das lagoas, e estas, menor que as dos açudes. Esta diferença pode ser explicada pelas características individuais de cada tipo de manancial. Os açudes e lagoas apresentam águas mais estáticas e com características próprias. Por exemplo, a composição das águas de cada açude será determinada pela localização deste último em relação à área da bacia que converge em sua direção, pelo volume de água que armazena, pelo tipo de solo e vegetação em volta, etc. Já nos rios, por apresentarem águas correntes, as variações entre si não são tão marcantes.

O resultado da análise da variação, para todo o conjunto de dados, mostrou-se altamente significativo a $1 \%$ para $\mathrm{Ca}$ e $\mathrm{Mg}$, significativo a $5 \%$ para $\mathrm{CE}, \mathrm{N}-\mathrm{NH}_{4}$, Fe e Si e não-significativo para $\mathrm{pH}, \mathrm{N}$ $\mathrm{NO}_{3}, \mathrm{P}, \mathrm{K}, \mathrm{Na}, \mathrm{Zn}$ e Mn. A comparação das médias entre açudes, lagoas e rios (Tabela 2) demonstra que houve diferença significativa entre mananciais para as variáveis: condutividade elétrica $(\mathrm{CE})$, teores de nitrogênio ( $\left.\mathrm{N}_{-} \mathrm{NH}_{4}, \mathrm{~N}-\mathrm{NO}_{3}\right), \mathrm{Ca}, \mathrm{Mg}$, Fe e Si. Os valores de $\mathrm{pH}$ e os teores de $\mathrm{P}, \mathrm{K}, \mathrm{Na}, \mathrm{Zn}$ e $\mathrm{Mn}$ não diferiram entre mananciais. Com exceção do $\mathrm{pH}$, os elevados coeficientes de variação, principalmente de $\mathrm{P}, \mathrm{Na}$ e $\mathrm{Mn}$, evidenciam a variação dos resultados entre os mananciais hídricos e explicam o porquê de os teores médios destes nutrientes não apresentarem diferenças significativas, embora numericamente os valores sejam distintos. A CE não diferiu entre os rios e açudes; porém, ambos foram menores que nas lagoas. Os valores mais elevados de CE nas lagoas da Região Sul do RS podem estar relacionados às maiores concentrações de $\mathrm{Na}$, $\mathrm{Ca}$ e $\mathrm{Mg}$ nessas águas, em relação aos demais mananciais analisados. Esta diferença pode ser explicada através da dinâmica dos elementos no solo, pois elementos com maior mobilidade, como $\mathrm{Na}, \mathrm{Ca}$, $\mathrm{Mg}$ e K, são mais facilmente lixiviados, enquanto outros, como o $\mathrm{P}$, que pela alta reatividade que, apresenta com os óxidos de $\mathrm{Fe}$ e Al, é mais fortemente adsorvido. Como na Região Sul, a lagoa é normalmente o destino final de todas as águas procedentes da bacia hidrográfica, ela tende a acumular as águas dos rios e açudes, além do fato, já mencionado, de que algumas lagoas recebem esporadicamente água do mar.

Os teores de $\mathrm{N}-\mathrm{NH}_{4}, \mathrm{~N}-\mathrm{NO}_{3}$ e Si foram mais elevados nos rios quando comparados às lagoas. Os teores de $\mathrm{Ca}$ e $\mathrm{Mg}$ foram maiores nas lagoas, sendo que o teor de $\mathrm{Mg}$ destas não diferiu dos rios. O teor de Fe foi menor nas lagoas quando comparado aos rios e açudes.

Os valores médios de $\mathrm{pH}$ obtidos neste trabalho (Tabela 2) estão dentro da variação de $\mathrm{pH}$ encontrada por MACEDO et al. (2003b,c) e acima dos valores encontrados por DESCHAMPS et al. (2003a) em águas de diferentes rios do RS e de SC, respectivamente. Os teores de $\mathrm{P}$ e $\mathrm{K}$ foram menores do que os encontrados em águas de rios, barragens e lagoas de outras regiões do Estado (MACEDO et al.,2001a, b; MARCHEZAN et al.,2002; MACEDO et al., 2003 a, b, c).

Os teores médios de Ca nas águas dos mananciais da Região Sul do RS foram semelhantes aos encontrados em águas de rios de outras regiões do RS e de SC (MACEDO et al., 2001a e b; MARCHEZAN et al., 2002; e DESCHAMPS et al.,

Tabela 2 - Teores médios de nutrientes $\left(\mu \mathrm{g} \mathrm{L}^{-1}\right), \mathrm{pH}$ e condutividade elétrica $\left(\mathrm{CE}, \mu \mathrm{S} \mathrm{cm}^{-1}\right)$ nas águas de açudes, lagoas e rios da Região Sul do RS (Média de vinte locais).

\begin{tabular}{|c|c|c|c|c|c|}
\hline \multirow{2}{*}{ Parâmetros } & \multicolumn{3}{|c|}{ Mananciais } & \multirow[b]{2}{*}{ Média } & \multirow[b]{2}{*}{ CV (\%) } \\
\hline & Açudes & Lagoas & Rios & & \\
\hline $\mathrm{pH}$ & $6,7 \mathrm{a}$ & $7,0 \mathrm{a}$ & $6,7 \mathrm{a}$ & 6,8 & 8 \\
\hline $\mathrm{CE}$ & $79 b$ & $174 a$ & $93 b$ & 116 & 91 \\
\hline $\mathrm{N}-\mathrm{NH}_{4}$ & $99 \mathrm{ab}$ & $70 \mathrm{~b}$ & $143 a$ & 104 & 72 \\
\hline $\mathrm{N}-\mathrm{NO}_{3}$ & $94 \mathrm{ab}$ & $69 b$ & $133 \mathrm{a}$ & 99 & 91 \\
\hline $\mathrm{P}$ & $27 \mathrm{a}$ & $35 \mathrm{a}$ & $60 \mathrm{a}$ & 41 & 146 \\
\hline K & $864 a$ & $1118 \mathrm{a}$ & $906 a$ & 963 & 73 \\
\hline $\mathrm{Ca}$ & $3133 c$ & $8130 a$ & $5790 \mathrm{~b}$ & 5684 & 57 \\
\hline $\mathrm{Mg}$ & $934 b$ & $1516 a$ & $1221 \mathrm{ab}$ & 1224 & 42 \\
\hline $\mathrm{Na}$ & $4480 \mathrm{a}$ & $7821 \mathrm{a}$ & $4974 a$ & 5760 & 118 \\
\hline $\mathrm{Zn}$ & $83 a$ & $89 a$ & $65 \mathrm{a}$ & 79 & 50 \\
\hline $\mathrm{Fe}$ & $1660 \mathrm{a}$ & $1330 \mathrm{~b}$ & $1631 \mathrm{a}$ & 1540 & 28 \\
\hline $\mathrm{Mn}$ & $290 \mathrm{a}$ & $185 \mathrm{a}$ & $219 a$ & 231 & 137 \\
\hline $\mathrm{Si}$ & $3782 \mathrm{ab}$ & $2772 b$ & $5046 a$ & 3870 & 64 \\
\hline
\end{tabular}

Médias seguidas da mesma letra, nas linhas, não diferem significativamente entre si pelo Teste de Duncan $(\mathrm{P}<0,05)$.

Ciência Rural, v.37, n.1, jan-fev, 2007. 
2003a). Da mesma forma, os teores médios de $\mathrm{N}\left(\mathrm{NH}_{4} \mathrm{e}\right.$ $\mathrm{NO}_{3}$ ) mantiveram-se próximos aos de outras regiões do RS e de SC (FURTADO et al., 2001; MACEDO et al., 2001a e b; MARCHEZAN et al., 2002; Macedo et al., 2003a, b e c; DESCHAMPS et al., 2003a). Os valores mais elevados de $\mathrm{N}-\mathrm{NO}_{3}$ nos rios podem estar relacionados às altas cargas de dejetos urbanos $\mathrm{e}$ industriais lançadas nos seus cursos, as quais estariam contribuindo para aumentar os teores desta forma de $\mathrm{N}$ na água. Os valores médios de $\mathrm{N}$ deste trabalho são semelhantes aos obtidos em pesquisa realizada na Região Sul, sobre a bacia hidrográfica das lagoas dos Patos e Mirim (MATTOS, 2004).

Os teores de $\mathrm{Mg}$ são semelhantes e, por vezes, menores, enquanto os teores de Fe estão de acordo com as variações apresentadas por outros pesquisadores (MACEDO et al., 2001a e b; MARCHEZAN et al., 2002; DESCHAMPS et al., 2003a). Os teores médios de $\mathrm{Si}$ foram semelhantes aos encontrados em águas para irrigação de arroz de rios e barragens da Índia (SAVANT et al.,1997)

A possível contribuição da água de irrigação de açudes, lagoas e rios da Região Sul do RS para a nutrição da cultura do arroz foi calculada com base no uso médio de água de $8000 \mathrm{~m}^{3} \mathrm{ha}^{-1} \mathrm{safra}^{-1}$ (GOMES et al., 2004) e nas concentrações mínimas (LI) e máximas (LS) do intervalo de confiança da média (IC a 5\%) (Tabelas 1). Os aportes mínimos e máximos estimados para cada elemento, em cada tipo de manancial hídrico, são apresentados na tabela 3 .

Os aportes de $\mathrm{Na}$, Si e $\mathrm{Ca}$ foram maiores, nos três mananciais, seguidos por $\mathrm{Fe}, \mathrm{K}$ e $\mathrm{Mg}$. A contribuição estimada de $\mathrm{Na}$ das águas das lagoas para as lavouras de arroz foi de, aproximadamente, o dobro da dos açudes e dos rios. Os aportes de Si pela água de irrigação variaram de 15 a $51 \mathrm{~kg} \mathrm{ha}^{-1}$, com média em $33 \mathrm{~kg}$ $\mathrm{ha}^{-1}$. Embora esta quantidade represente, por exemplo, apenas $4 \%$ do total acumulado $\left(890 \mathrm{~kg} \mathrm{ha}^{-1}\right)$ pela cultivar "IR8" (YOSHIDA, 1981), em termos absolutos, parece ser suficiente para o suprimento deste elemento, assumindo-o necessário como micronutriente, sem contar ainda a contribuição do solo onde é abundante.

$\mathrm{O}$ aporte de potássio variou de $3,7 \mathrm{a} 11 \mathrm{~kg}$ $\mathrm{ha}^{-1}$, sendo maior nas lagoas. Estes valores foram inferiores ao aporte de $48 \mathrm{~kg} \mathrm{ha}^{-1}$ estimado para $\mathrm{K}$ pelas águas de irrigação do Rio Gravataí (MACEDO et al., 2003c). Considerando-se que uma lavoura de arroz remove quantidades de $\mathrm{K}$ em torno de $309 \mathrm{~kg} \mathrm{ha}^{-1}$ (YOSHIDA, 1981), os aportes deste nutriente, pela água de irrigação da Região Sul do RS, representariam apenas 1,2 a $3,6 \%$ do total acumulado pela cultura. Convém ressaltar, contudo, que esta forma de comparação e discussão de dados constitui apenas um exercício, uma vez que as quantidades removidas pela cultura podem variar conforme o clima, o tipo de solo, os níveis de nutrientes no solo, a cultivar e a produtividade (FAGERIA, 1984). Por exemplo, se o mesmo cálculo anterior for feito considerando-se um acúmulo menor de $\mathrm{K}$, em torno de $95 \mathrm{~kg} \mathrm{ha}^{-1}$ (FAGERIA, 1984), o aporte deste nutriente pelas águas de irrigação da Região Sul do RS representaria de 4 a $12 \%$ do potássio extraído pelas plantas, alterando, portanto, a magnitude de seu significado. Além destes fatores, o cálculo do aporte pode mudar também em função da variação no consumo de água.

$\mathrm{O}$ aporte médio de Ca foi estimado em $65 \mathrm{~kg}$ $\mathrm{ha}^{-1}$ nas lagoas, seguido pelos rios, com $47 \mathrm{~kg} \mathrm{ha}^{-1}$, e pelos açudes, com $25 \mathrm{~kg} \mathrm{ha}^{-1}$. Tais valores são bastante

Tabela 3 - Estimativa do aporte de nutrientes pela água de irrigação de açudes, lagoas e Rios da Região Sul do RS, durante o ciclo da cultura do arroz, considerando um uso médio de água de $8000 \mathrm{~m}^{3} \mathrm{ha}^{-1}$ safra ${ }^{-1}$ e as concentrações mínimas e máximas do intervalo de confiança da média $(5 \%)$.

\begin{tabular}{|c|c|c|c|c|c|c|}
\hline \multirow{2}{*}{ Nutriente } & \multicolumn{2}{|c|}{ Açudes } & \multicolumn{2}{|c|}{ Lagoas } & \multicolumn{2}{|c|}{ Rios } \\
\hline & mínimo & máximo & mínimo & máximo & mínimo & máximo \\
\hline & \multicolumn{6}{|c|}{$\mathrm{kg} \mathrm{ha}^{-1}$} \\
\hline $\mathrm{N}-\mathrm{NH}_{4}$ & 0,56 & 1,02 & 0,45 & 0,68 & 0,74 & 1,6 \\
\hline $\mathrm{N}-\mathrm{NO}_{3}$ & 0,38 & 1,14 & 0,30 & 0,81 & 0,70 & 1,43 \\
\hline $\mathrm{P}$ & 0,11 & 0,32 & 0,16 & 0,40 & 0,13 & 0,82 \\
\hline $\mathrm{K}$ & 3,7 & 10 & 7,0 & 11 & 4,8 & 10 \\
\hline $\mathrm{Ca}$ & 18 & 32 & 49 & 81 & 35 & 58 \\
\hline $\mathrm{Mg}$ & 5,3 & 9,6 & 10 & 14 & 8,3 & 11 \\
\hline $\mathrm{Na}$ & 15 & 57 & 25 & 101 & 32 & 48 \\
\hline $\mathrm{Zn}$ & 0,51 & 0,82 & 0,57 & 0,85 & 0,38 & 0,67 \\
\hline $\mathrm{Fe}$ & 11 & 16 & 10 & 11 & 12 & 14 \\
\hline $\mathrm{Mn}$ & 0,44 & 4,20 & 0,78 & 2,20 & 1,30 & 2,20 \\
\hline $\mathrm{Si}$ & 20 & 40 & 15 & 29 & 30 & 51 \\
\hline
\end{tabular}


significativos, uma vez que a quantidade de $\mathrm{Ca}$ removida pela cultura é em torno de $27 \mathrm{~kg}$ por hectare (YOSHIDA, 1981), indicando que, na Região Sul do $\mathrm{RS}$, este nutriente poderia ser totalmente ou quase suprido pelas águas de irrigação. Este fato também foi constatado por MACEDO et al. (2001b) e MARCHEZAN et al. (2002) para outras regiões do Estado.

Os aportes médios estimados de $\mathrm{Mg}(9,6 \mathrm{~kg}$ ha $\left.^{-1}\right)$ e de Fe (13,5kg ha-1), em relação aos teores desses elementos extraídos pela cultura do arroz irrigado, $34,8 \mathrm{~kg} \mathrm{ha}^{-1}$ e $4,8 \mathrm{~kg} \mathrm{ha}^{-1}$, respectivamente, (YOSHIDA, 1981 ), representariam $27,6 \%$ do $\mathrm{Mg}$ e mais que o dobro (281\%) do Fe acumulados pelas plantas.

Os aportes médios de $\mathrm{N}\left(2,2 \mathrm{~kg} \mathrm{ha}^{-1}\right)$ e $\mathrm{P}$ $\left(0,47 \mathrm{~kg} \mathrm{ha}^{-1}\right)$ pelas águas dos rios da Região Sul foram menores que os estimados por MACEDO et al. (2003c) para as águas de irrigação do Rio Gravataí $\left(80 \mathrm{~kg} \mathrm{~N} \mathrm{ha}^{-1} \mathrm{e}\right.$ $0,7 \mathrm{~kg} \mathrm{P} \mathrm{ha}^{-1}$ ). Como foi utilizado o mesmo consumo de água em ambos os cálculos, as diferenças podem ser explicadas pelas maiores concentrações de $\mathrm{N}$ e $\mathrm{P}$ no Rio Gravataí, decorrentes do grande volume de resíduos lançados nas suas águas, em razão da sua proximidade a centros urbanos e industriais (MACEDO et al., 2001b). As contribuições médias estimadas de N e P pelas águas de irrigação da Região Sul do RS, quando comparadas com as quantidades acumuladas de $164 \mathrm{~kg} \mathrm{ha}^{-1}$ de $\mathrm{Ne}$ de $46 \mathrm{~kg} \mathrm{ha}^{-1}$ de P (YOSHIDA, 1981) representam apenas $1,3 \%$ do $\mathrm{N}$ e $1,0 \%$ do $\mathrm{P}$ removidos pela cultura do arroz irrigado,

$\mathrm{Na}$ adubação NPK da cultura do arroz irrigado no sistema de semeadura em solo seco, as doses recomendadas para os estados do RS e de SC variam de 40 a $120 \mathrm{~kg} \mathrm{ha}^{-1}$ de N, de 20 a $90 \mathrm{~kg} \mathrm{ha}^{-1}$ de P e de 20 a $80 \mathrm{~kg} \mathrm{ha}^{-1}$ de $\mathrm{K}$, levando-se em conta a porcentagem de matéria orgânica, os teores de fósforo e de potássio do solo e a expectativa de rendimento da cultura (CQFS-NRS, 2004). Os aportes de NPK pelas águas de irrigação dos mananciais analisados na Região Sul do RS (Tabela 3) representam, em relação à atual recomendação de adubação do arroz irrigado, para o $\mathrm{N}, 0,5$ a 1,8\%; para o P, 0,5 a 2,3\% e, para o K, 9,3 a 37\%. $\mathrm{O}$ aporte mais significativo de $\mathrm{K}$ confirma as constatações de outros pesquisadores, que atribuíram a baixa resposta da cultura do arroz irrigado à adubação com este nutriente à contribuição através da água de irrigação (MACHADO, 1985; LOPES, 1991; MACEDO et al., 2001b).

Os dados obtidos nesta pesquisa, juntamente com os de outros mananciais hídricos, fontes de captação de água para irrigação da cultura do arroz no Estado do Rio Grande do Sul, poderão servir de subsídio para a melhor compreensão dos fatores que influem na resposta à adubação do arroz irrigado por inundação.

\section{CONCLUSÕES}

Na média, os elementos presentes nas águas de açudes, lagoas e rios utilizados para irrigação de arroz na Região Sul do Rio Grande do Sul decrescem na seqüência $\mathrm{Na}>\mathrm{Ca}>\mathrm{Si}>\mathrm{Fe}>\mathrm{Mg}>\mathrm{K}>\mathrm{Mn}>\mathrm{N}>\mathrm{Zn}$ $>$ P. Os teores desses elementos diferem entre os mananciais, sendo que os teores de N, Fe e Si são mais elevados nos rios, que nas lagoas; os de $\mathrm{Ca}$ e $\mathrm{Mg}$ nas lagoas, do que nos açudes; e os de Fe nos açudes e rios, do que nas lagoas. Os aportes de nutrientes estimados através de suas concentrações nas águas de irrigação evidenciam que essas quantidades correspondem ao que, em média, é acumulado, nas plantas de arroz, de cálcio, ferro, silício e sódio e de até $28 \%$ de magnésio e $12 \%$ de potássio.

\section{REFERÊNCIAS}

COMISSÃO DE QUÍMICA E FERTILIDADE DO SOLO-RS/ SC. Manual de adubação e de calagem para os Estados do Rio Grande do Sul e de Santa Catarina. 10.ed. Porto Alegre, SBCS-NRS. 2004. 394p.

DESCHAMPS, F. et al. A qualidade da água em áreas cultivadas com arroz irrigado. In: CONGRESSO BRASILEIRO DE ARROZ IRRIGADO, 3.; REUNIÃO DA CULTURA DO ARROZ IRRIGADO, 25., 2003, Balneário Camboriú, SC. Anais... Itajaí: Epagri, 2003a. p.700-702.

DESCHAMPS, F.C. et al. Aplicação de um índice de qualidade (IQA) na avaliação da água de áreas cultivadas com arroz irrigado. In: CONGRESSO BRASILEIRO DE ARROZ IRRIGADO, 3.; REUNIÃO DA CULTURA DO ARROZ IRRIGADO, 25., 2003, Balneário Camboriú, SC. Anais... Itajaí: Epagri, 2003; p706708 b.

FAGERIA, N.K. Adubação e nutrição mineral da cultura de arroz. Goiânia: EMBRAPA-CNPAF, 1984. 341p.

FURTADO, R.D. et al. Dinâmica ambiental de nutrientes na água durante o período de irrigação, em três técnicas de cultivo de arroz (Oriza sativa). In: CONGRESSO BRASILEIRO DE ARROZ IRRIGADO, 2.; REUNIÃO DA CULTURA DO ARROZ IRRIGADO, 24., 2001, Porto Alegre, RS. Anais... Porto Alegre: Instituto Rio grandense de Arroz, 2001. p.772-774.

GENRO Jr., S.A. et al. Contribuição de nutrientes pela água de irrigação do Rio Gravataí e da Barragem do Capané para a cultura do arroz irrigado. In: CONGRESSO BRASILEIRO DE ARROZ IRRIGADO, 4.; REUNIÃO DA CULTURA DO ARROZ IRRIGADO, 26., 2005, Santa Maria RS. Anais... Santa Maria: Editora Orium, 2005. p502-504.

GOMES, A.S. et al. Uso e manejo de água em arroz irrigado. In: GOMES, A.S.; MAGALHÃES Jr., A.M. Arroz irrigado no Sul do Brasil. Brasília, DF: Embrapa Informação Tecnológica, 2004. Cap. 14 , p.417-455

KORNDÖRFER, G.H. et al. Avaliação de métodos de extração de silício para solos cultivados com arroz de sequeiro. Revista Brasileira de Ciência do Solo, Viçosa, v.23, n.1, p101-106, 1999. 
LOPES, S.I.G. Eficiência da adubação potássica e distribuição radicular do arroz irrigado. 1991. $96 \mathrm{f}$. Dissertação (Mestrado em Agronomia - Solos) - Programa de Pós-graduação em Agronomia, Universidade Federal do Rio Grande do Sul, Porto Alegre.

MACEDO, V.R.M. et al. Perdas de solo e nutrientes na água de drenagem durante o preparo do solo para o sistema de cultivo de arroz pré-germinado. In: CONGRESSO BRASILEIRO DE ARROZ IRRIGADO, 2.; REUNIÃO DA CULTURA DO ARROZ IRRIGADO, 24., 2001, Porto Alegre, RS. Anais... Porto Alegre: Instituto Rio Grandense de Arroz, 2001a. p.247-249.

MACEDO, V.R.M.et al. Levantamento exploratório da composição química das águas utilizada para a irrigação do arroz no Rio Grande do Sul. In: CONGRESSO BRASILEIRO DE ARROZ IRRIGADO, 2.; REUNIÃO DA CULTURA DO ARROZ IRRIGADO, 24., 2001, Porto Alegre, RS. Anais... Porto Alegre: Instituto Rio Grandense de Arroz, 2001b. p793-795.

MACEDO, V.R.M. et al. Perdas de nutrientes e solo em diferentes manejos da água no sistema pré-germinado. In: CONGRESSO BRASILEIRO DE ARROZ IRRIGADO, 3; REUNIÃO DA CULTURA DO ARROZ IRRIGADO, 25., 2003, Balneário Camboriú, SC. Anais... Itajaí: EPAGRI, 2003a. p184-186.

MACEDO, V.R.M. et al. Nutrientes nas águas da bacia hidrográfica do Rio Vacacaí Mirim durante o período de cultivo de arroz irrigado. In: CONGRESSO BRASILEIRO DE ARROZ IRRIGADO, 3; REUNIÃO DA CULTURA DO ARROZ IRRIGADO, 25., 2003, Balneário Camboriú, SC. Anais... Itajaí: EPAGRI, 2003b. p218-220.

MACEDO, V.R.M. et al. Composição química da água do Rio Gravataí utilizada no campo experimental do IRGA em Cachoeirinha. In: CONGRESSO BRASILEIRO DE ARROZ IRRIGADO, 3.; REUNIÃO DA CULTURA DO ARROZ IRRIGADO, 25., 2003, Balneário Camboriú SC. Anais... Itajaí: EPAGRI, 2003c. p221-222.

MACEDO, V.R.M.et al. Características da água do reservatório da Barragem do Arroio Duro usado para irrigação da lavoura de arroz. In: CONGRESSO BRASILEIRO DE ARROZ IRRIGADO,
4., REUNIÃO DA CULTURA DO ARROZ IRRIGADO, 26., 2005, Santa Maria RS. Anais... Santa Maria: Orium, 2005. p.484-486.

MACHADO, M.O. Caracterização e adubação do solo. In: EMBRAPA-CPATB. Fundamentos para a cultura do arroz irrigado. Campinas: Fundação Cargill, 1985. p.129-179.

MATTOS M.L.T. A cultura do arroz irrigado e o meio ambiente. In: GOMES, A. da S.; MAGALHÃES Jr., A.M. Arroz irrigado no Sul do Brasil. Brasília, DF: Embrapa Informação Tecnológica, 2004. Cap.25, p.861-899.

MARCHEZAN, E. et al. Perda de nutrientes na água de drenagem inicial na cultura do arroz irrigado. In: CONGRESSO DA CADEIA PRODUTIVA DE ARROZ, 1., REUNIÃO NACIONAL DE PESQUISA DE ARROZ, 7., 2002, Florianópolis, SC. Anais... Santo Antônio de Goiás: Embrapa Arroz e Feijão, 2002. p.680683.

PINTO, L.F.S.et al. Solos de várzea do Sul do Brasil cultivados com arroz irrigado. In: GOMES, A. da S.; MAGALHÃES Jr., A.M. Arroz irrigado no Sul do Brasil. Brasília, DF: Embrapa Informação Tecnológica, 2004. Cap.3, p.75-95.

SAVANT, N.K. et al. Silicon management and sustainable rice production. Advances in Agronomy, New York, v.58, p.151199,1997

YOSHIDA, S. Fundamentals of rice crop science. Los Baños, Philippines: International Rice Research Institute, 1981. 269p.

TEDESCO, M.J et al. Análises de solo, plantas e outros materiais. 2.ed. Porto Alegre: Departamento de Solos, UFRGS, 1995. 174p.

VAHL, L.C. Nutrição de plantas de arroz irrigado. In: PESKE, S.T. et al. Produção de arroz irrigado. 3.ed. Pelotas: Universidade Federal de Pelotas, 2004. p1.53-202.

ZONTA, E.P.; MACHADO, A.A. SANEST - Sistema de análise estatística. Pelotas: Departamento de Matemática, Estatística e Computação da UFPel, 1984. 151p. 